\title{
QUASI-EQUICONTINUOUS SETS OF FUNCTIONS ${ }^{1}$
}

\section{CHIEN WENJEN}

The well-known theorem of analysis that if $F$ is a family of functions defined, equicontinuous, and uniformly bounded on a bounded closed set $E$ in $n$-dimensional real cartesian space $R^{n}$, then from every sequence $\left\{f_{n}\right\}$ of functions of $F$ it is possible to select a uniformly convergent subsequence, has been recently generalized to various abstract spaces $[1 ; 4 ; 6]$. Consider a set $F$ of continuous functions on one topological space $X$ to another, $Y$. For any point $x$ of $X$ and any open set $W$ of $Y$ we denote by $(x, W)$ the totality of functions $f$ in $F$ for which $f(x) \in W$. The topology in $F$ obtained by employing all sets of the $(x, W)$ as a subbase in $F$ is called the $p$-topology by Arens [2]. The purpose of this note is to find the necessary and sufficient conditions that it be possible to select a subsequence converging pointwise to a continuous function from any given sequence of continuous functions and the necessary and sufficient conditions that a set of continuous functions be compact in the $p$-topology.

Definition. Let $\left\{f_{n}\right\}$ be a sequence of functions from a topological space $X$ to be metric space $Y .\left\{f_{n}\right\}$ is said to be $\epsilon$-related at a point $x \in X$ if for every arbitrarily chosen $\epsilon>0$ there is a neighborhood $U(x)$ of $x$ such that, corresponding to each point $x^{\prime} \in U(x)$, a positive number $N_{\epsilon}\left(x, x^{\prime}\right)$ can be determined satisfying the condition:

$$
\rho\left[f_{n}(x), f_{n}\left(x^{\prime}\right)\right]<\epsilon
$$

whenever $n>N_{\epsilon}\left(x, x^{\prime}\right)$.

Definition. Let $F$ be a family of continuous functions from a topological space $X$ to a metric space $Y . F$ is called quasi-equicontinuous if in every infinite subset $Q$ of $F$ and at any point $x \in X$ there is a sequence $\left\{f_{n}\right\}$ contained in $Q$ which is $\epsilon$-related at $x$.

THEOREM. If $X$ is locally separable and $Y$ metric, a set of functions $F \subset Y^{X}$, where $Y^{\boldsymbol{X}}$ denotes the set of all continuous functions from $X$ to $Y$, is compact under p-topology if and only if

(1) $F$ is closed in $Y^{x}$,

(2) $F(x)=\bigcup_{f \in F} f(x)$ is compact for every $x \in X$,

(3) $F$ is quasi-equicontinuous.

Presented to the Society, February 26, 1955; received by the editors December 31, 1954 and, in revised form, April 11, 1955.

1 This problem was suggested by Professor Richard Arens to whom the writer is indebted for many valuable suggestions. 
Proof. I. Necessity. (1) $F$ is closed since $F$ is compact and $Y^{x}$ is a Hausdorff space under $p$-topology.

(2) Let $g_{x}(f)=f(x)$. Then $g_{x}$ is a continuous function of $f$ and the compactness of $F(x)$ follows from the compactness of $F$.

(3) Since compactness implies countable compactness, any infinite subset $Q$ of $F$ has a limit point $f$ contained in $F$. Let $\left\{x_{n}\right\}$ be an enumerable set contained and dense in a neighborhood $U\left(x_{0}\right)$ of a point $x_{0}$ in $X$. We can find a subset $\left\{f_{n}\right\}$ of $Q$ satisfying

$$
\begin{aligned}
& \rho\left[f_{n}\left(x_{0}\right), f\left(x_{0}\right)\right]<1 / n, \\
& \rho\left[f_{n}\left(x_{1}\right), f\left(x_{1}\right)\right]<1 / n, \\
& \cdot \cdot \cdot \cdot \cdot \cdot \cdot \cdot, \\
& \rho\left[f_{n}\left(x_{n}\right), f\left(x_{n}\right)\right]<1 / n, \quad n=1,2,3, \cdots .
\end{aligned}
$$

Then

$$
f_{n}\left(x_{k}\right) \rightarrow f\left(x_{k}\right), \quad k=1,2,3, \cdots
$$

as $n \rightarrow \infty$.

Next we show that $f_{n}(x) \rightarrow f(x)$ at any point $x$ in $U\left(x_{0}\right)$. Suppose on the contrary that $f_{n}(x)$ does not converge to $f(x)$ at certain point $x^{\prime}$ in $U\left(x_{0}\right)$. There exist an $\epsilon>0$ and a subsequence $\left\{f_{n_{i}}\right\}$ of $\left\{f_{n}\right\}$ such that

$$
\rho\left[f_{n_{i}}\left(x^{\prime}\right), f\left(x^{\prime}\right)\right]>\epsilon, \quad i=1,2,3, \cdots .
$$

Let $g$ be a limit point of $\left\{f_{n_{i}}\right\}$ in $F$ and let a subsequence $\left\{f_{n^{\prime} i}\right\}$ of $\left\{f_{n_{i}}\right\}$ be so chosen that

$$
\begin{aligned}
& \rho\left[f_{n_{i}^{\prime}}\left(x^{\prime}\right), g\left(x^{\prime}\right)\right]<1 / i, \\
& \rho\left[f_{n_{i}^{\prime}}\left(x_{1}\right), g\left(x_{1}\right)\right]<1 / i, \\
& \cdot \cdot \cdot \cdot \cdot \cdot \cdot \cdot \cdot, \\
& \rho\left[f_{n_{i}}\left(x_{i}\right), g\left(x_{i}\right)\right]<1 / i, \quad i=1,2,3, \cdots .
\end{aligned}
$$

Then

$$
\begin{aligned}
f_{n^{\prime} i}\left(x^{\prime}\right) & \rightarrow g\left(x^{\prime}\right), \\
f_{n^{\prime} i}\left(x_{k}\right) & \rightarrow g\left(x_{k}\right), \quad k=1,2,3, \cdots,
\end{aligned}
$$

as $n_{i}^{\prime}$ approaches to infinity. Now it is clear that

$$
\lim f_{n^{\prime} i}\left(x_{k}\right)=\lim f_{n_{i}}\left(x_{k}\right)=f\left(x_{k}\right)=g\left(x_{k}\right), \quad k=1,2,3, \cdots .
$$

We have therefore

$$
f(x)=g(x)
$$


for all $x$ in $U\left(x_{0}\right)$ on account of the continuity of the functions $f(x)$ and $g(x)$. It follows that

$$
f_{n_{i}^{\prime}}\left(x^{\prime}\right) \rightarrow g\left(x^{\prime}\right)=f\left(x^{\prime}\right) .
$$

The contradiction between the relations (A) and (B) proves that $f_{n}(x)$ converges to $f(x)$ at any point $x$ in $U\left(x_{0}\right)$. In other words,

$$
\lim _{n \rightarrow \infty} \lim _{x \rightarrow x_{0}} f_{n}(x)=\lim _{x \rightarrow x_{0}} \lim _{n \rightarrow \infty} f_{n}(x) .
$$

Hence $\left\{f_{n}\right\}$ is $\epsilon$-related at $x_{0} .{ }^{2}$ The quasi-equicontinuity of the set of functions $F$ is proved.

II. Sufficiency. Since $F(x)$ is compact for any $x \in X$, the topological product $G=\prod_{x \in X} F(x)$ is compact. Consider the correspondence between $F$ and a subset $S$ of $G$ obtained by assigning to each $f \in F$ the point in $G$ with coordinates $f(x), x$ ranging over $X$; this correspondence is a homeomorphism. In order to prove the compactness of $F$ it is sufficient to prove that $S$ is compact, that is, to prove that $S$ is closed in $G$.

Let $g$ be a limit point of $S$ with coordinates $g(x)$. There exists a sequence $\left\{f_{n}\right\} \subset F$ such that

$$
\begin{aligned}
& \rho\left[f_{n}\left(x_{0}\right), g\left(x_{0}\right)\right]<1 / n, \\
& \rho\left[f_{n}\left(x_{1}\right), g\left(x_{1}\right)\right]<1 / n, \\
& \cdot \cdot \cdot \cdot \cdot \cdot \cdot \cdot, \\
& \rho\left[f_{n}\left(x_{n}\right), g\left(x_{n}\right)\right]<1 / n, \quad n=1,2,3, \cdots,
\end{aligned}
$$

where $\left\{x_{n}\right\}$ is an enumerable set dense in a neighborhood $U\left(x_{0}\right)$ of $x_{0}$. By the quasi-equicontinuity of the set of functions $F$ there is a subsequence $\left\{f_{n_{i}}\right\}$ of $\left\{f_{n}\right\}$ such that for each $\epsilon>0$ there is a neighborhood $V\left(x_{0}\right)$ of $x_{0}$ contained in $U\left(x_{0}\right)$ such that

$$
\rho\left[f_{n_{i}}\left(x_{0}\right), f_{n_{i}}(x)\right]<\epsilon
$$

for any $x \in V\left(x_{0}\right)$ and all $n_{i}>N_{\epsilon}\left(x_{0}, x\right)$. Now

$$
\begin{gathered}
\rho\left[g\left(x_{0}\right), g\left(x_{k}\right)\right]<\rho\left[g\left(x_{0}\right), f_{n_{i}}\left(x_{0}\right)+\rho\left[f_{n_{i}}\left(x_{0}\right), f_{n_{i}}\left(x_{k}\right)\right]\right. \\
+\rho\left[f_{n_{i}}\left(x_{k}\right), g\left(x_{k}\right)\right]<3 \epsilon
\end{gathered}
$$

for any $x_{k} \in V\left(x_{0}\right)$, if $n_{i}$ is sufficiently large. By the same reasoning for any point $x$ in $V\left(x_{0}\right)$ there is a neighborhood $W(x)$ of $x$ contained in $U\left(x_{0}\right)$ such that

2 That the $\epsilon$-related condition was given by Hobson as a necessary and sufficient condition for interchange of order in repeated limits was pointed out by the referee [6, p. 409]. 


$$
\rho\left[g(x), g\left(x_{k}\right)\right]<3 \epsilon \quad \text { if } \quad x_{k} \in\left\{x_{n}\right\} \cap W(x) .
$$

Then

$$
\rho\left[g\left(x_{0}\right), g(x)\right]<\rho\left[g\left(x_{0}\right), g\left(x_{j}\right)\right]+\rho\left[g\left(x_{j}\right), g(x)\right]<6 \epsilon
$$

for any $x \in V\left(x_{0}\right)$, where $x_{j} \in V\left(x_{0}\right) \cap W(x) . g(x)$ is therefore continuous at $x_{0}$, that is, $g$ belongs to $S$ and the closedness of $S$ is proved.

Corollary. Let $F$ be a family of continuous functions from a separable space $X$ to a metric space $Y$. The necessary and sufficient conditions that it be possible to select a subsequence converging pointwise to a continuous function from any given sequence of functions of $F$ are:

(1) $F(x)$ is countably compact for any $x \in X$,

(2) $F$ is quasi-equicontinuous.

Corollary. Let $(C)$ be the set of all continuous functions defined on the closed interval $(0,1)$ and let $F$ be any subset of $(C) . F$ is weakly compact if and only if it is weakly closed and quasi-equicontinuous.

\section{REFERENCES}

1. R. Arens, $A$ topology for spaces of transformations, Ann. of Math. vol. 47 (1946) pp. $480-495$.

2. - Duality in linear spaces, Duke Math. J. vol. 14 (1947) pp. 787-794.

3. E. F. Eberlein, Weak compactness in Banach space I, Proc. Nat. Acad. Sci. U.S.A. vol. 33 (1947) pp. 51-53.

4. D. Gale, Compact sets of functions and function rings, Proc. Amer. Math. Soc. vol. 1 (1950) pp. 303-308.

5. E. W. Hobson, The theory of functions of a real variable and the theory of Fourier's series, vol. 1, 3d ed.

6. S. B. Myers, Equicontinuous sets of mappings, Ann. of Math. (2) vol. 47 (1946) pp. 496-502.

University of California, Los Angeles 